\title{
The Changing Face and Focus of the Adolescent with an Eating Disorder
}

\author{
Carly Chason*, Krista M. Davis, Lynae J. Hanks and Krista Casazza
}

\begin{abstract}
University of Alabama at Birmingham, Department of Pediatrics, Division of General Pediatrics and Adolescent Medicine, $16014^{\text {th }}$ Ave S, Children's Park Place I 310, Birmingham, AL, 35233, USA
\end{abstract}

\begin{abstract}
The classic silhouette of the typical adolescent with an eating disorder (ED) is a white female with extremely low body weight; this has begun to take a new shape. The most apparent shift in the classic views are sex and weight. A trend toward a progressively decreasing age of onset in ED has also emerged. The objective of this paper is to describe the ED patients presenting to Children's of Alabama's Adolescent Eating Disorders Clinic, encompassing their age, sex, BMI percentile and muscle function. The data was examined from all first presentations to the clinic from 2013 to 2016. Between May 2013 and March 2016, 102 new patient appointments were scheduled. 88 patient's charts were reviewed from the time of each initial appointment in the Adolescent Eating Disorders Clinic to obtain the sex, age, race, height, weight, reason for referral/active problems and ED diagnosis for each patient. BMI percentile was calculated according to reference ranges for sex and age. Handgrip strength was measured by dynamometer. As is consistent with previously published data, there were significantly more females than males seen in our population. There were more females than males across all categories of ED diagnoses. The highest number of diagnoses occurred between the ages of 13-16. A positive correlation between BMI percentile and measured handgrip strength was observed. Measured handgrip strength in females was lower in ages 13-18 than expected grip strength for age. When males were grouped by "Males 14 and under" and "Males 15 and over," a lower measured grip strength compared to the expected grip strength for age was demonstrated. The impact of ED on morbidity and mortality has been well recognized; however the most often reported association was mainly based on changes in body weight. The adverse metabolic consequences perturb nutrient sensing and ultimately delivery and utilization. A shift in the focus of energy balanced towards systemic malnourishment may allow healthy and sustained metabolic improvements.
\end{abstract}

Keywords: Adolescent, eating disorder, muscle function, handgrip strength, metabolic health.

\section{INTRODUCTION}

The classic silhouette of the typical adolescent with an eating disorder (ED) is a white female with extremely low body weight; this has begun to take a new shape. While current reports espousing the classic view such as that documented in primary care may be aligned with overall estimates [1], a broader and more comprehensive assessment may reveal the changing face of ED in this decade. For example, Loth et al. [2] recently reported that a national survey of adolescents in the United States indicated that $21.8 \%$ of girls and $11.2 \%$ of boys had self-reported engaging in disordered eating behaviors within the last 30 days. Consideration of shifts in the current dogmatic view of ED and subsequent treatment may provide better strategies for optimizing growth and development during this critical stage.

The most apparent shift in the classic views are those encompassing sex and weight. Although the changes in the DSM-V criteria make the diagnosis of anorexia nervosa (AN) applicable to males, there have been changes in the prevalence rates of ED among

*Address correspondence to this author at the University of Alabama at Birmingham, Department of Pediatrics, Division of General Pediatrics and Adolescent Medicine, $16014^{\text {th }}$ Ave S, Children's Park Place I 310, Birmingham, AL, 35233, USA; Tel: 205-934-4531; Fax: 205-975-6503;

E-mail: kcasazza@peds.uab.edu males prior to those changes. A 2009 study in Australia found that $24.7 \%$ of adolescents with early onset eating disorders were male $[3,4]$. Shifts in weight to include those within the normal $\mathrm{BMI}$ range (i.e. $20^{\text {th }}<\mathrm{BMI}$ percentile $<85^{\text {th }}$ ) are also apparent. While adolescents suspected of an ED have most likely experienced substantial weight loss over a relatively short period of time with consequent malnourishment, a normal body mass index (BMI) percentile may limit initiation of an ED diagnosis. It was recently reported among inpatient admissions for AN, the proportion of those who were not underweight rose nearly $40 \%$ from 2008 to 2014 [5]. Concomitantly, the appearance of adequate fat mass among these individuals may mask the loss of skeletal muscle mass and function elicited by nutrient deficiencies as well as cardiac anomalies [6]. Thus, despite being atypical in the context of our inherent perceptions of ED, increased awareness of the changing face is requisite.

Furthermore, there appears to be a trend toward a progressively decreasing age of onset in $E D$, with the median age of onset being 12.3 years old [7]. A bimodal increase in eating disorders, one prepubertally and one post-pubertally (average age of onset (combining two modes) 15-19) has been noted; however most research has been conducted in patients older than 15 years (post-pubertal) and nearly all in females. 
The objective of this paper is to describe the ED patients presenting to Children's of Alabama's Adolescent Eating Disorders Clinic, encompassing their age, sex, BMI percentile and muscle function.

\section{METHODS}

\section{Setting}

The Adolescent Eating Disorders Clinic is a specialty clinic offered through the Adolescent Health Center at Children's Hospital of Alabama that provides comprehensive inter-disciplinary outpatient care for adolescents. Encompassed by the mission of the Adolescent Health Center in which it is housed, Eating Disorders clinic provides services for adolescents ages 11-21 with eating disorders. All patients referred to the clinic were evaluated by a nurse, registered dietitian, and pediatrician. The pediatrician performed medical evaluation including reproductive maturity and diagnosis of ED. The dietitian gathered information on diet, physical activity and feeding behaviors.

\section{Sample and Procedure}

The data was examined from all first presentations to the clinic from 2013 to 2016. Between May 2013 and March 2016, 102 new patient appointments were scheduled. Of those 102 scheduled appointments, 88 patients presented for their initial appointments (i.e. the additional 14 made appointments but did not show up for their appointment). Patients were excluded from data analysis if they either cancelled or did not appear for their appointment. 88 patient's charts were reviewed from the time of each initial appointment in the Adolescent Eating Disorders Clinic to obtain the sex, age, race, height, weight, reason for referral/active problems and ED diagnosis for each patient. BMI percentiles and Z-scores were calculated using the Pediatric Z-Score Calculator from the Children's Hospital of Philadelphia Research Institute website. DSM-V criteria was used to assign ED diagnosis. Malnourishment was determined based on weight loss over the past three months, physical findings of pediatrician, presence of wasting and/or biochemical assessment.

Table 1 presents the demographic characteristics of the 88 patients that were reviewed. The majority of the population was female $(85.2 \%)$, and the mean age of the population was 14.4 years. Patients in the ages 15 and $16(40.9 \%)$ were comparable in number to those ages 13 and 14 (37.5\%). The remaining patients were comparably distributed in ages 11 and 12 (11.4\%), and
17 and $18(9.1 \%)$, with 1 in the 8 to 10 age group (1.1\%). The population was primarily non-Hispanic white $(79.5 \%)$, with $9.1 \%$ non-Hispanic black, 9.1\% Hispanic, and $2.3 \%$ classified as other.

Table 1: Population Demographics at the Adolescent Eating Disorders Clinic as Determined by Electronic Medical Record Review for Patients Presenting during 2013-2016

\begin{tabular}{|c|c|c|c|}
\hline Characteristics & Category & N & $\%$ \\
\hline \hline \multirow{2}{*}{ Sex } & Male & 13 & 14.8 \\
\cline { 2 - 4 } & Female & 75 & 85.2 \\
\hline \multirow{2}{*}{ Age (yrs) } & $<15$ & 44 & 50 \\
\cline { 2 - 4 } & $>15$ & 44 & 50 \\
\hline \multirow{2}{*}{ Race } & Caucasian & 70 & 79.5 \\
\cline { 2 - 4 } & African American & 8 & 9.1 \\
\cline { 2 - 4 } & Hispanic & 8 & 9.1 \\
\cline { 2 - 4 } & Other & 2 & 2.3 \\
\hline
\end{tabular}

\section{Measures}

Height, weight and age were collected for the population, with BMI percentile and BMI Z-score calculated for each patient. The mean BMI Z-score was -0.71 with the mode being -2.26 .

The Children's of Alabama's Adolescent Eating Disorders Clinic receives referrals from both health care providers, including physicians and psychologists, and parents. There were six reasons for referrals for this population: malnutrition, disordered eating, weight loss, weight concerns, restrictive eating, vomiting, and solid food refusal.

Table 2: Reasons for Referral of Adolescents to Eating Disorders Clinic

\begin{tabular}{|c|c|c|}
\hline Reason for Referral & N & \% \\
\hline \hline Malnutrition & 46 & 52.8 \\
\hline Disordered eating & 5 & 5.7 \\
\hline Weight loss & 26 & 29.9 \\
\hline Weight concerns & 1 & 1.1 \\
\hline Restrictive eating & 4 & 4.6 \\
\hline Vomiting & 4 & 4.6 \\
\hline Solid food refusal & 1 & 1.1 \\
\hline
\end{tabular}

In the ED population, many of the same diagnoses are seen across patients. The most common diagnostic codes observed in this population relate to weight loss 
and malnutrition. Other frequent diagnostic codes include overweight/obesity and abnormal weight gain, as well as other various diagnoses that are also prevalent in this population. Of the 88 patients reviewed, diagnoses related to disordered eating included all of the following:

Vomiting (self-induced), problems related to inappropriate diet and eating habits, weight loss, underweight, abnormal weight gain, abnormal weight loss, malnutrition, unspecified nutritional deficiency, laxative abuse, severe protein-calorie malnutrition, persistent vomiting, overweight, moderate malnutrition, bulimia nervosa, anorexia nervosa (restricting type), morbid obesity due to excess kcal, conversion disorder with motor symptom or deficit, binge eating (purging type)

Following the change in diagnostic criteria for eating disorders from DSM-IV to DSM-V, we see a wider variety of diagnoses among the population from 2013 to 2016. However, the diagnoses can continue to be broken into three subsets of eating disorders: AN, BN, and other specified feeding or eating disorder (OSFED). OSFED encompasses atypical anorexia nervosa, atypical bulimia nervosa, binge-eating disorder, purging disorder, and night eating syndrome. The breakdown of these diagnoses for this population is illustrated in Figure 1.

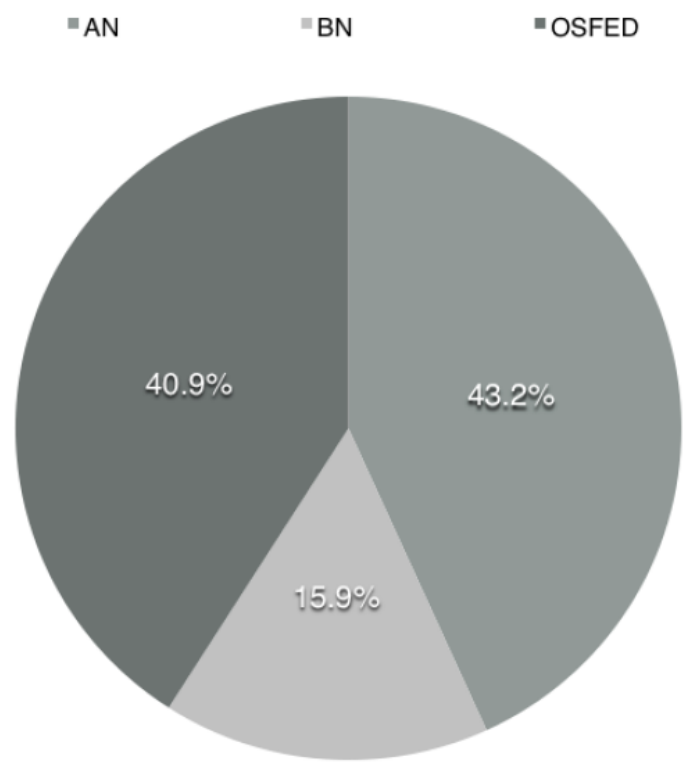

Figure 1: Diagnoses of eating disorders for population.

For a subset of the population, handgrip strength was assessed using a dynamometer in pounds per square inch (PSI). Results were classified using normative data for age and sex and are reported in Figure 5.

\section{RESULTS}

In order to gain a further understanding of the population, differences in diagnoses between subsets of the population based on sex, age group, and race were assessed. As is consistent with previously published data, there were significantly more females than males seen in our population. As illustrated in Figure 2, there were more females than males across all categories of ED diagnoses. The highest number of diagnoses occurred between the ages of $13-16$ as shown in Figure 3. This figure also demonstrates the span of eating disorders from ages 8 to 18. Measured handgrip strength in females was lower in ages 13-18 than expected grip strength for age as shown in Figure 5; the outliers illustrated in this figure are the higher

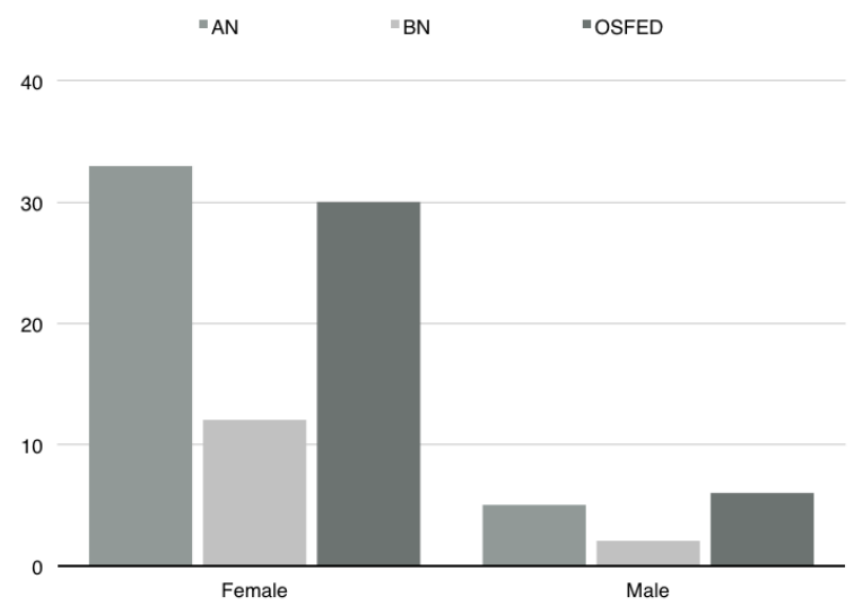

Figure 2: Diagnoses for females vs. males.

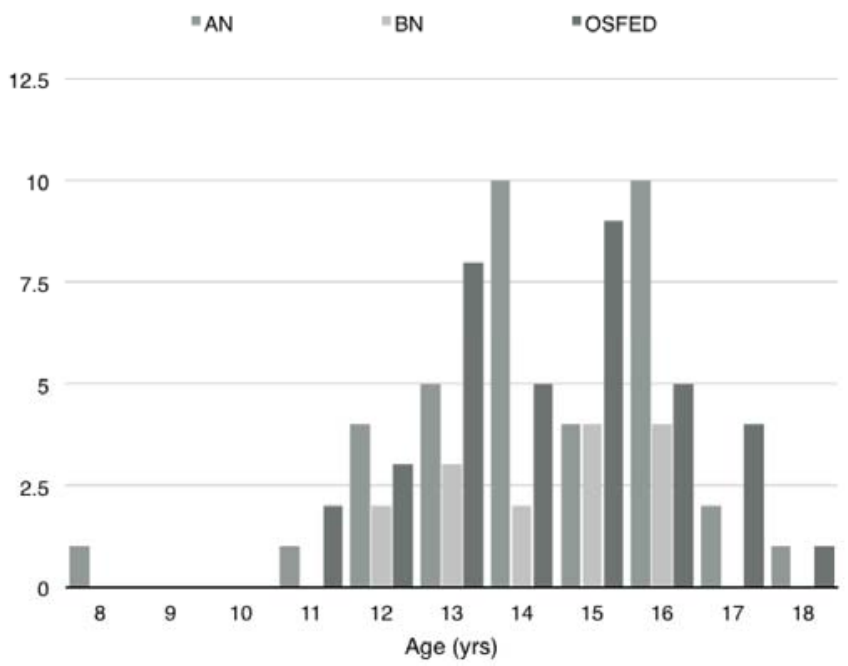

Figure 3: Diagnoses for age. 
measured grip strengths in females ages 11 and 12 compared to expected grip strength for these ages. When males were grouped by "Males 14 and under" and "Males 15 and over," a lower measured grip strength compared to the expected grip strength for age was demonstrated. A positive correlation is presented in Figure 7 between BMI percentile and measured handgrip strength.
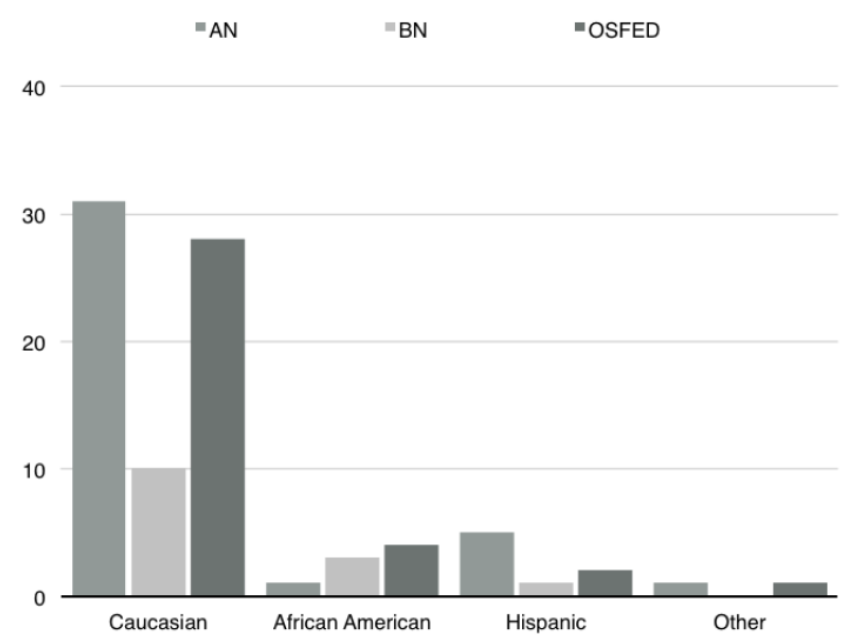

Figure 4: Diagnoses for races.

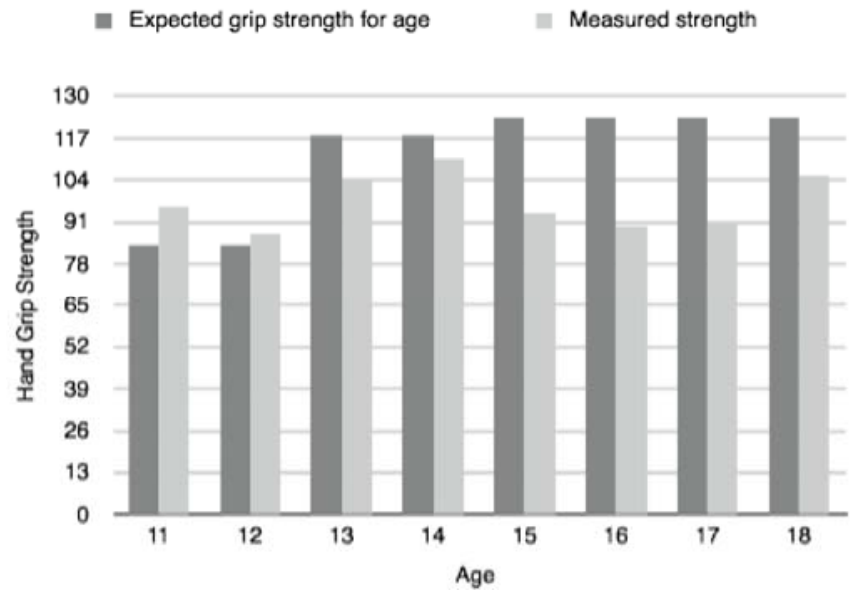

Figure 5: Comparison between expected grip strength and measured grip strength for age in females. Expected grip strengths are derived from reference data for adolescent females.

\section{DISCUSSION}

This descriptive study demonstrates the face of the "typical" ED patient has become atypical. As the face of ED in the adolescent population changing a strategic shift in the balance of our energy in treatment is requisite. Important physiologic and psychologic complications are likely to manifest even in the absence of overt thinness. The majority of patients continue to be non-Hispanic White females with AN

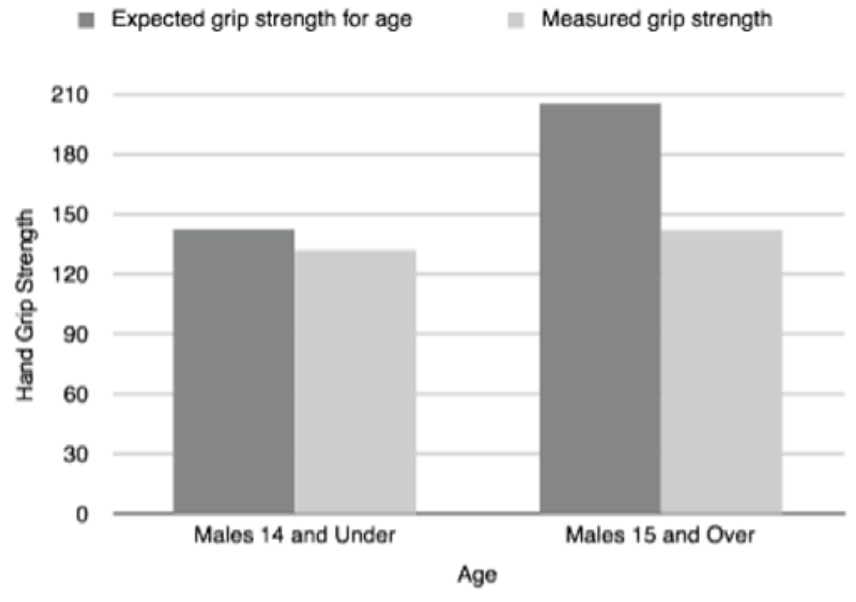

Figure 6: Comparison between expected and measured grip strengths in males. Expected grip strengths are derived from reference data for adolescent males.

diagnoses. However, this is significantly lower than the oft report $\sim 90 \%$ in many reports [8]. We illustrate the a noticeable increase in diagnoses in males, racial/ethnic minorities and importantly OSFED, indicating that despite significant weight loss, the individual's weight is within or above normal range. For the males in our population, OSFED diagnoses were the most prevalent, closely followed by $\mathrm{AN}$, which is a new diagnoses for males following the updated DSM-V. For those whose disorders fall within this category, the time to diagnosis is frequently much longer than a patient with a "classic" ED [9], highlighting the need for increased awareness across disciplines. These patients not only have the same spectrum of behaviors and conditions, characteristics of typical ED patients, but also had similar physiologic complications extending across body systems. Measured hand grip strength fell below the expected grip strengths for age and sex, indicating impaired musculoskeletal function. In addition, as noted by [6]. These adolescents also often present with bradycardia, despite not being underweight. The extent of physical compromise reported here demonstrates considerable impact on health during this critical period of physiologic and psychologic development.

BMI percentiles of our population spanned the continuum, highlighting the occurrence of malnourishment despite normal weight. While malnutrition is frequently recognized in ED patients, it is often reported in the context of body fat and/or weight. Besides biomechanical function, skeletal muscle is capable of synthesizing and releasing factors that modulate bone metabolism. Decreased contractility leads to loss of strength, fatigue, etc., prior to overt muscle wasting [10]. In addition to nutritional and 


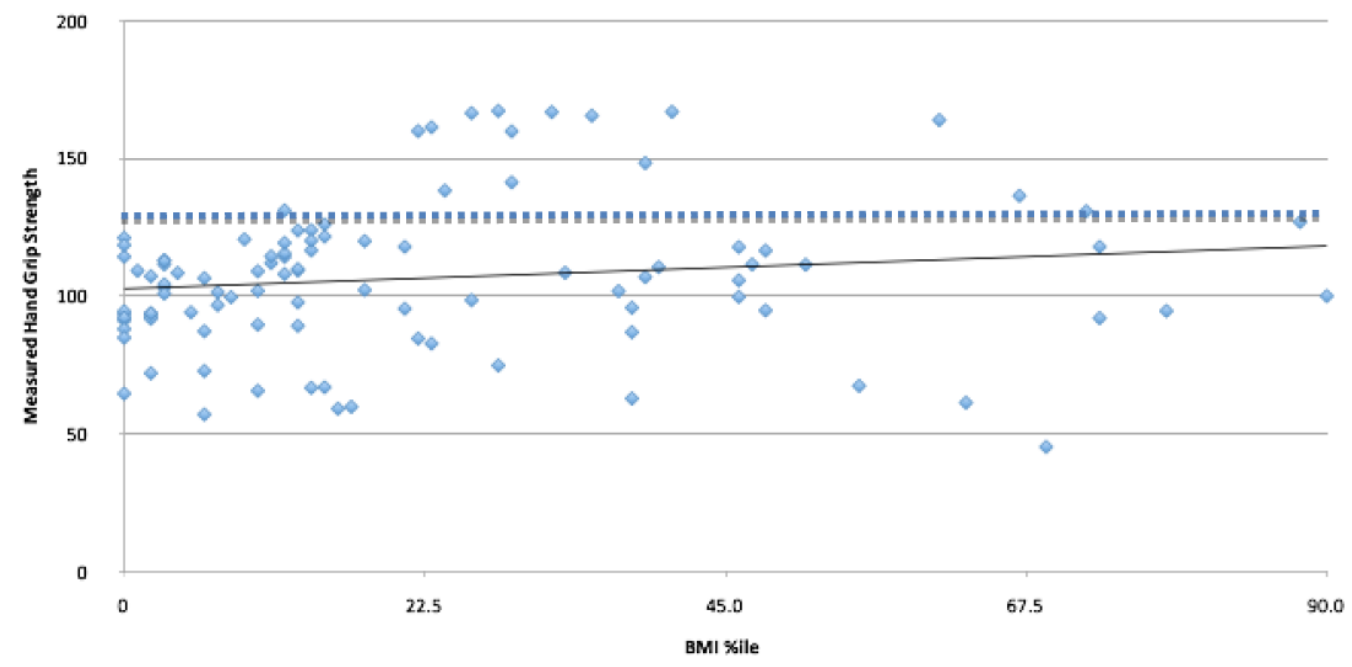

Expected

Figure 7: Correlation between BMI \%ile and measured hand grip strength.

physical activity factors associated with the musclebone relationship, as evidenced by chronic diseases, systematic inflammation can directly induce muscle and bone loss $[11,12]$. By recording an objective measure of skeletal muscle function, strategies can be implemented to preserve, maintain, or increase skeletal muscle mass.

A strength of this review is that all patient data assessed was from current referrals and appointments, all within the past three years. Additionally, all diagnoses fall under the new DSM-V criteria, providing a more appropriate representation of the contemporary ED population. Limitations include diagnostic criteria having been determined from medical record notes from the ED Clinic at Children's of Alabama, as opposed to official diagnoses from mental health professionals, who typically provide an ED diagnosis. In order to continue assessing the ED populations, further exploration of system wide clinical manifestations of malnourishment in adolescents without chronic medical conditions is an area of research that would likely provide additional insight into changes in the prevalence and patterns of ED.

The impact of ED on morbidity and mortality has been well recognized; however the most often reported association was mainly based on changes in body weight. In catabolic conditions, skeletal muscle releases amino acids to support protein synthesis in other tissues such as liver, immune cells and intestinal mucosa. In anabolic conditions, the non-essential amino acid glutamine is largely produced by de novo synthesis and stored in skeletal muscle. The adverse metabolic consequences perturb nutrient sensing and ultimately delivery and utilization. A shift in the focus of energy balanced towards systemic malnourishment may allow healthy and sustained metabolic improvements.

\section{REFERENCES}

[1] Herpertz-Dahlmann B. Adolescent Eating Disorders: Definitions, Symptomatology, Epidemiology and Comorbidity. Child and Adolescent Psychiatric Clinics of North America 2009; 18(1): 31-47. http://dx.doi.org/10.1016/i.chc.2008.07.005

[2] Loth K, MacLehose R, Bucchianeri M, Crow S, NeumarkStainer D. Personal and Socio-environmental predictors of dieting and disordered eating behaviors from adolescence to young adulthood: 10 year longitudinal findings 2014.

[3] Madden S, Morris A, Zurynski YA, Kohn M, Elliot EJ. Burden of eating disorders in 5-13 year-old children in Australia. Med J Aust 2009; 190(8): 410-14.

[4] Ackard DM, Fulkerson JA, Neumark-Sztainer D. Prevalence and utility of DSM-IV eating disorder diagnostic criteria among youth. Int J Eat Disord 2007; 40(5): 409-17. http://dx.doi.org/10.1002/eat.20389

[5] Whitelaw M, Gilbertson H, Lee KJ, Sawyer SM. Restrictive eating disorders among adolescent inpatients. Pediatrics 2014; 134(3): e758. http://dx.doi.org/10.1542/peds.2014-0070

[6] Sawyer SA, Whitelaw M, Le Grange D, Yeo M, Hughes EK. Physical and Psychological Morbidity in Adolescents with Atypical Anorexia Nervosa. Pediatrics 2016; 137(4): e20154080.

[7] Swanson SA, Crow SJ, Le Grange D, Swendsen J, Merikangas KR. Prevalence and correlates of eating disorders in adolescents: Results from the National Comorbidity Survey Replication Adolescent Supplement. Arch Gen Psychiatry 2011; 68(7): 714-23. http://dx.doi.org/10.1001/archgenpsychiatry.2011.22

[8] Merikangas KR, He J, Burstein M, Swanson SA, Avenevoli S, Cui L, et al. Lifetime prevalence of mental disorders in U.S. adolescents: Results from the National Comorbidity StudyAdolescent Supplement (NCS-A). J Am Acad Child Adolesc Psychiatry 2010; 49(10): 980-89. http://dx.doi.org/10.1016/j.jaac.2010.05.017

[9] Peebles R, Hardy KK, Wilson JL, Lock JD. Medical compromise in eating disorders not otherwise specified: Are diagnostic criteria for eating disorders markers of severity? Pediatrics 2010; 125(5): e1193-e1201. 
[10] Westmoreland P, Krantz MJ, Mehler PS. Medical complications of Anorexia Nervosa and Bulimia. The American Journal of Medicine 2016; 129(1): 30-37. http://dx.doi.org/10.1016/j.amjmed.2015.06.031

[11] Cianferrotti L, Brandi ML. Muscle-bone interactions: basic and clinical aspects. Endocrine 2014; 45(2): 165-77. http://dx.doi.org/10.1007/s12020-013-0026-8
[12] Robinson L, Aldridge V, Clark EM, Mirsa M, Micali M. A systematic review and meta-analysis of the association between eating disorders and bone density. Osteoporosis International 2016; 27(6): 1953-66.

http://dx.doi.org/10.1007/s00198-015-3468-4 